\title{
Impact of Electronic Medication Management on the Physical Location of Work in a Paediatric Setting
}

\author{
Bethany A VAN DORT ${ }^{\mathrm{a}, \mathrm{b}, 1}$, Melissa T BAYSARI ${ }^{\mathrm{a}, \mathrm{b}}$, Mirela PRGOMET ${ }^{\mathrm{b}}$, Wu Yi \\ ZHENG $^{\mathrm{a}, \mathrm{b}}$, Magdalena Z RABAN ${ }^{\mathrm{b}}$, Luciano DALLA-POZZA ${ }^{\mathrm{c}}$, Cheryl \\ MCCULLAGH ${ }^{\mathrm{d}}$ and Johanna WESTBROOK ${ }^{\mathrm{b}}$ \\ ${ }^{a}$ The University of Sydney, Faculty of Medicine and Health, Australia \\ ${ }^{\mathrm{b}}$ Australian Institute of Health Innovation, Macquarie University, Australia \\ ' Cancer Centre for Children, The Children's Hospital at Westmead, Australia \\ d'The Sydney Children's Hospital Network, Australia
}

\begin{abstract}
Electronic medication management (eMM) systems can have a significant impact on efficiency and safety. There is limited evidence on the effects of eMM implementation on the physical location of work. The objective of this study was to evaluate the impact of eMM and associated hardware implementation on the location of tasks performed by doctors and nurses. 41.5 hours of observation were conducted in the oncology ward of a paediatric hospital. Tasks, locations and resources used were recorded pre and post eMM implementation. Results showed that a wider variety of locations were used to conduct tasks following eMM implementation. Post-eMM, more tasks were performed in the hallway, where medication trolleys with attached laptops were situated, and in patient rooms where additional computers were installed, providing more opportunities for patient/carer and clinician interaction. The findings from this study reveal the impact that computer placement has on the location of work for doctors and nurses, and the importance of planning hardware placement for eMM implementation.
\end{abstract}

Keywords. Medical order entry systems, computers, implementation, observational study.

\section{Introduction}

Implementation of electronic medication management (eMM) systems in hospitals can lead to a reduction in medication errors, increased legibility of documentation and improved accessibility of information for users [1-3]. Studies evaluating the impact of eMM in paediatric hospitals have also found a reduction in adverse drug events and patient mortality following system implementation $[4,5]$.

With the implementation of eMM, many tasks that were traditionally done on paper in the ward can now be completed on a computer from any location. Qualitative studies exploring user perceptions following eMM implementation suggest that access to the system from computers located anywhere in the hospital was considered beneficial $[6,7]$.

${ }^{1}$ Bethany A Van Dort, The University of Sydney, Charles Perkins Centre, John Hopkins Drive, Camperdown NSW 2006, Australia. Email: bethany.vandort@sydney.edu.au 
When investigating the impact of eMM implementation on clinician workflow, previous studies have focused on work efficiency [1] or errors and patient safety [2, 3, $8,9]$. To our knowledge, there have been no studies investigating the impact of eMM implementation on the physical location of work in the paediatric setting. This study aimed to determine how introduction of an eMM system impacted on the location of work tasks performed by doctors and nurses.

\section{Method}

\subsection{Study design and setting}

This study used a before and after study design and a non-participatory observational approach. Observations of doctors and nurses were conducted in a 20-bed oncology ward at a paediatric hospital in Sydney, Australia. Pre-eMM observations were conducted between March and June 2016 and post-eMM observations between August and October, 2018. Ethics approval was obtained from the Sydney Children's Hospital Network Human Research Ethics Committee (LNR/15/SCHN/171).

Prior to eMM implementation, the hospital had an existing clinical information system in place for pathology and imaging orders and results. Patient medication charts, progress/clinical notes, fluid balance charts, observation charts and chemotherapy protocols were paper-based and typically stored in folders in hallway workstations located outside each patient room. Computers were accessed via a central workstation. An eMM system and electronic patient notes were implemented in the oncology ward in August 2016 and electronic observation charts in April 2017. To facilitate access to the new system, the ward acquired medication trolleys with laptop computers, wall-mounted computers in all patient rooms, and a computer in the medication room.

\subsection{Participants and procedure}

Nurses and doctors were recruited opportunistically on the inpatient oncology ward. Five researchers with previous qualitative research experience independently shadowed doctors and nurses for a maximum of 2 hours at a time. Researchers recorded notes during observations with a focus on medication-related tasks that involved access to information or documentation. Researchers met periodically to discuss their observations and code findings into categories: actors, tasks, locations, and resources used. Observations ceased when saturation was reached. In total, 3 doctors and 6 nurses were observed pre-eMM implementation for a total observation time of 26 hours and 15 minutes. 5 doctors and 6 nurses were observed post-eMM implementation for a total of 15 hours and 15 minutes.

\section{Results}

Pre-eMM implementation, most tasks performed by doctors were conducted at the hallway workstation. Post-eMM implementation, doctors no longer conducted work in the hallway workstation, instead they conducted medication-related tasks from their office, the central workstation, or the computer in the patient's room (Table 1). 
Table 1. Tasks and locations of information tasks performed by doctors before and after eMM implementation

\begin{tabular}{|c|c|c|c|c|c|}
\hline \multirow[t]{2}{*}{ Task } & \multicolumn{5}{|c|}{ Location } \\
\hline & Meeting room & $\begin{array}{c}\text { Hallway } \\
\text { workstation* }\end{array}$ & Doctors' office & $\begin{array}{c}\text { Central } \\
\text { workstation }\end{array}$ & Patient room \\
\hline Ward round & $\ulcorner\square$ & & & & \\
\hline $\begin{array}{l}\text { Review medications/ } \\
\text { observations/ fluids }\end{array}$ & & 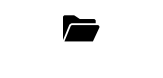 & $\square$ & $\square$ & $\square$ \\
\hline $\begin{array}{l}\text { Prescribe/ cease/ edit } \\
\text { medications }\end{array}$ & & $\sigma$ & $\square$ & $\square$ & $\square$ \\
\hline $\begin{array}{l}\text { Review and record } \\
\text { progress notes }\end{array}$ & & $\digamma$ & $\square$ & $\square$ & $\square$ \\
\hline $\begin{array}{l}\text { Execute plans (e.g. } \\
\text { order tests, follow up) }\end{array}$ & & & $\sqsubset \square$ & $\square$ & $\square$ \\
\hline
\end{tabular}

indicates Pre-eMM and $\square$ indicates Post-eMM; *Hallway workstation: pre-eMM implementation this was a folder stored outside the patient's room, post-eMM implementation medication trolleys with attached laptop computers were kept in the hallway.

Table 2. Tasks and locations of information tasks performed by nurses before and after eMM implementation

\begin{tabular}{|c|c|c|c|c|c|c|}
\hline \multirow[t]{2}{*}{ Task } & \multicolumn{6}{|c|}{ Location } \\
\hline & $\begin{array}{l}\text { Meeting } \\
\text { room }\end{array}$ & $\begin{array}{c}\text { Hallway } \\
\text { workstation* }\end{array}$ & $\begin{array}{c}\text { Central } \\
\text { workstation }\end{array}$ & $\begin{array}{l}\text { Medication } \\
\text { room }\end{array}$ & $\begin{array}{l}\text { Cytotoxic } \\
\text { room }\end{array}$ & $\begin{array}{c}\text { Patient } \\
\text { room }\end{array}$ \\
\hline Ward round & $\ulcorner\square$ & & & & & \\
\hline Check observations & & & & & & $\nabla \square$ \\
\hline $\begin{array}{l}\text { Review and record } \\
\text { observations and } \\
\text { fluids }\end{array}$ & & $\ulcorner\square$ & & & & $\square$ \\
\hline $\begin{array}{l}\text { Administer } \\
\text { medication }\end{array}$ & & & & & & $\sqsubset \square$ \\
\hline Review medications & & $\Gamma \square$ & $\ulcorner\square$ & & & $\square$ \\
\hline Prepare medications & & $\square$ & & $\ulcorner\square$ & & \\
\hline $\begin{array}{l}\text { Prepare } \\
\text { chemotherapy }\end{array}$ & & & & & $\sigma \square$ & \\
\hline $\begin{array}{l}\text { Double-check } \\
\text { medications }\end{array}$ & & $\square$ & & $\ulcorner\square$ & $\sqsubset \square$ & $\square$ \\
\hline Review pathology & & $\square$ & $\sqcap \square$ & & & $\square$ \\
\hline $\begin{array}{l}\text { Review and record } \\
\text { progress notes }\end{array}$ & & $\square$ & $\ulcorner\square$ & & & $\square$ \\
\hline $\begin{array}{l}\text { Check medication } \\
\text { guidelines }\end{array}$ & & $\square$ & $\ulcorner\square$ & $\ulcorner\square$ & & \\
\hline
\end{tabular}

$\approx$ indicates Pre-eMM and $\square$ indicates Post-eMM ; *Hallway workstation: pre-eMM implementation this was a folder stored outside the patient's room, post-eMM implementation medication trolleys with attached laptop computers were kept in the hallway. 
Similarly, pre-eMM nurses conducted many tasks at the hallway workstation and central workstation, but post-eMM, these tasks were conducted from multiple locations (Table 2). For example, reviewing and recording of observations and fluids pre-eMM were only completed at the hallway workstation, however post-eMM they were also done on the computer at the patient bedside. Pre-eMM medication preparation was only done in the medication room, however, post-eMM implementation, it was also conducted in the hallway using medication trolleys with attached laptop computers.

For both doctors and nurses, there was also an increase in the types of tasks that were conducted from the patient room using wall mounted computers.

\section{Discussion}

Implementation of eMM was associated with significant shifts in where doctors and nurses performed their work. The majority of task types that were largely conducted at the hallway workstation pre-eMM were conducted in a greater variety of locations posteMM. For both doctors and nurses, many task types after eMM implementation were performed at the patient bedside, where previously, they were only performed in the hallway or central workstations. Introduction of medication trolleys with attached laptop computers resulted in some medication preparation by nurses being done in the hallway post-eMM as opposed to only the medication room.

For both doctors and nurses, computers at the patient bedside meant more tasks could be undertaken in patients' rooms. Previous Australian studies have found eMM implementation led to doctors spending more time with patients [1] and found that nurses have a preference for conducting tasks in patient rooms [10]. Conversely, a study in an emergency department found doctors and nurses spent less time on direct patient care post-eMM implementation, most likely because computers were positioned in a designated 'charting room', not in patient rooms[11]. Although we did not quantify the time spent on tasks in patient rooms, computers in patient rooms gave doctors and nurses more opportunities to perform work in those locations, providing patients/carers and clinicians with more opportunities to interact. Qualitative studies with patients and parents have found increased clinician communication and establishing interpersonal relationships to be important aspects of paediatric hospital care [12-14]. Consequently, providing doctors and nurses with more opportunities to interact with patients/carers, by placing computers in patient rooms, is likely to positively impact care for paediatric patients.

The infrastructure implemented with the eMM system allowed more flexibility in where medication-related tasks that required documentation and access to information, could be performed. Post-eMM implementation, we observed more variability in the locations of medication-related work, with almost all tasks conducted in multiple locations. Determining whether this increased variability was positive or negative for staff and patients was outside the scope of our study, but we recommend future evaluations of the placement of hardware on clinical workflow after eMM implementation.

This study was conducted in the oncology ward of a single paediatric hospital which may limit the applicability of results to other institutions. As this was an observational study, there is potential bias due to the Hawthorn effect [15], that is, participants may have changed their behaviours as a result of being observed. However, we expect this impact to be minimal as the information we collected related to tasks performed, 
resources used and locations, and clinicians were aware that no assessments or evaluations of quality were being made. It is also important to note that workload and time taken to complete tasks were not measured, and patient experiences of the change in work location were not explored.

To conclude, this study showed the impact eMM implementation and placement of computers had on the location of doctors' and nurses' work. The increase we observed in the types of tasks performed in patients' rooms has potential positive implications for patients/carers, particularly in the paediatric setting, due to the value they place on interactions with clinicians. Future hospital implementations should evaluate computer placement choices to make the best decisions for clinical workflow specific to their department.

\section{References}

[1] Westbrook JI, Li L, Georgiou A, et al. Impact of an electronic medication management system on hospital doctors' and nurses' work: a controlled pre-post, time and motion study. Journal of the American Medical Informatics Association 20 (2013), 1150-58.

[2] Prgomet M, Li L, Niazkhani Z, et al. Impact of commercial computerized provider order entry (CPOE) and clinical decision support systems (CDSSs) on medication errors, length of stay, and mortality in intensive care units: a systematic review and meta-analysis. Journal of the American Medical Informatics Association 24 (2016), 413-22.

[3] Qureshi NA, Al-Dossari DS, Al-Zaagi IA, et al. Electronic health records, electronic prescribing and medication errors: A systematic review of literature, 2000-2014. Journal of Advances in Medicine and Medical Research (2015), 672-704.

[4] Longhurst CA, Parast L, Sandborg CI, et al. Decrease in Hospital-wide Mortality Rate After Implementation of a Commercially Sold Computerized Physician Order Entry System. Pediatrics 126 (2010), 14.

[5] Upperman JS, Staley P, Friend K, et al. The impact of hospitalwide computerized physician order entry on medical errors in a pediatric hospital. Journal of Pediatric Surgery 40 (2005), 57-59.

[6] Baysari MT, Hardie R-A, Lake R, et al. Longitudinal study of user experiences of a CPOE system in a pediatric hospital. International Journal of Medical Informatics 109 (2018), 5-14.

[7] Cresswell KM, Bates DW, Williams R, et al. Evaluation of medium-term consequences of implementing commercial computerized physician order entry and clinical decision support prescribing systems in two 'early adopter' hospitals. Journal of the American Medical Informatics Association 21 (2014), e194-e202.

[8] Gates PJ, Meyerson SA, Baysari MT, et al. The Prevalence of Dose Errors Among Paediatric Patients in Hospital Wards with and without Health Information Technology: A Systematic Review and MetaAnalysis. Drug Safety 42 (2019), 13-25.

[9] Chen AR, Lehmann CU. Computerized provider order entry in pediatric oncology: design, implementation, and outcomes. Journal of Oncology Practice 7 (2011), 218-22.

[10] Andersen P, Lindgaard A-M, Prgomet M, et al. Mobile and Fixed Computer Use by Doctors and Nurses on Hospital Wards: Multi-method Study on the Relationships Between Clinician Role, Clinical Task, and Device Choice. Journal of Medical Internet Research 11 (2009), e32.

[11] Park SY, Lee SY, Chen Y. The effects of EMR deployment on doctors' work practices: A qualitative study in the emergency department of a teaching hospital. International Journal of Medical Informatics 81 (2012), 204-17.

[12] Zwaanswijk M, Tates K, van Dulmen S, et al. Young patients', parents', and survivors' communication preferences in paediatric oncology: Results of online focus groups. BMC Pediatrics 7 (2007), 35.

[13] Coyne I, Gallagher P. Participation in communication and decision-making: children and young people's experiences in a hospital setting. Journal of Clinical Nursing 20 (2011), 2334-43.

[14] Fisher MJ, Broome ME. Parent-Provider Communication During Hospitalization. Journal of Pediatric Nursing 26 (2011), 58-69.

[15] McCambridge J, Witton J, Elbourne DR. Systematic review of the Hawthorne effect: New concepts are needed to study research participation effects. Journal of Clinical Epidemiology 67 (2014), 267-77. 